\title{
Design and Implementation of Crop Potential Model System Based on GIS and Componentware Technology
}

\author{
Hao Zhang ${ }^{1}$, Li Ding ${ }^{1}$, Guang Zheng ${ }^{1}$, Xin Xu ${ }^{1}$, Lei Xi ${ }^{1, *}$, and Xinming $\mathrm{Ma}^{1,2}$ \\ ${ }^{1}$ College of Information and Management Science, Henan Agricultural University, \\ Zhengzhou 450002, China \\ ${ }^{2}$ College of Agronomy, Henan Agricultural University, Zhengzhou 450002, China \\ zhanghaohnnd@126.com, hnaustu@126.com
}

\begin{abstract}
Based on SuperMap IS.NET and the empirical models about crop potential output, the paper firstly designed the model system of crop potential output by using componentware method based on distributed computing architecture under network environment. Secondly, the paper implemented crop potential model components by using componentware technology. Finally, with the abstract mechanism of interface, the paper integrated crop potential output models and loosely coupled model components with SuperMap GIS. The results show that the model system as a component container about crop potential output model integrated empirical and mechanism models and provided a dynamic management for crop potential output models and dynamic methods call, which solved the issues of integration and expansion, and the system has the characteristics of wide applicability and good independence, which provides $\mathrm{ADM}$ and technical support for the construction of major grain-producing areas, crop production management and potential mining.
\end{abstract}

Keywords: Componentware, Crop potential output, GIS, UML.

\section{Introduction}

Currently, digital model on crop production system is the foundation and the core of the digital agriculture, and is also the bridge linking the planting digitization, intelligentization and precision[1-4]. The significance of crop potential output model lies in the quantitative analysis and evaluation for the factors' role to the whole crop producing stage, and explains the factors' impact to crop potential output in detail. Crop potential model has advantages of strong explanatory power, wide application and easy to quantify and be controlled.

With the development of crop potential output evaluation model, componentware and GIS, GIS-driven software development methods and componentware technology have been widely used in various types of GIS application system. It is effective to reduce the coupling GIS with model system and improve the maintainability and independence by using componentware technology[5-6]. In this case, the paper adopted the

\footnotetext{
* Corresponding author.
} 
componentware method to build a strong extension and low coupling crop potential model system based on SuperMap IS.NET 2008, accomplished crop production information management and potential analysis, and provided technical support and decision-making for managing crop production and mining crop potential.

\section{Data Source and Research Method}

\subsection{Data Source}

Research data includes meteorological data, soil data, socio-economic data and crop production data. Meteorological data includes daily average temperature, daily maximum temperature, daily minimum temperature, rainfall and sunshine hours, provided by the weather bureau of Henan Province. Soil information includes soil texture, soil type and soil nutrients, provided by the county soil station or compiled through Henan TuRang Dili[7]. Socio-economic data includes producing condition, economic condition and producing level. Crop production data includes crop varieties, planting region, annual crop area, yield per hectare, annual total output and multiple crop index, collected from the statistical yearbook of Henan Province.

\subsection{Research Method}

Crop Potential Output Evaluation Process. First, based on the process and the method of crop potential output evaluation[8-9], the paper collected and collated attribute data, such as Meteorology, soil and social production, and spatial data at 30 counties in Henan Province, to integrate space and attributes database. Second, the paper built the model components at all levels based on crop potential output model about light, temperature, water and soil and realized the model system of crop potential output. Finally, the paper quantitatively calculated crop potential output through mechanism model.

Crop Potential Output Mechanism Models. The frequent methods of crop potential output included mechanism model and empirical model[10-12] at present. The latter was complicated and had the disadvantage of more inputted parameters leading to be difficult to spread, but the former was simple and easy to popularize. So, the paper used mechanism model of light, temperature, water, soil and social-economic factors, designed crop potential output model components, and constructed a crop production potential model system. Crop potential output model included computing and analysis models of crop potential output. Computing model included natural and social resource calculation. Computing model of natural resource included solar radiation model, photosynthetic potential model, temperature potential model, climate potential model and soil potential model. Computing model of social resource was used to quantify social factors' contribution, such as producing condition, economic condition and producing level. The daily and total solar radiations were calculated during crop growth according to reference [13]. The potential output of photosynthesis, temperature, climate and soil was calculated according to reference [14-18]. Similarly, Analysis model of crop potential output included natural and social resource analysis, and the analysis approaches included spatial analysis and time analysis. 


\section{Design of Model System}

\subsection{System Architecture}

Crop potential output model system consisted of system tool, system data, potential calculation and potential evaluation. System tool was responsible for system data management and made up of data editing components and model components for generating meteorological data of previous and current years. System data included the input database and the output database. The input database was made up of regional data, meteorological data, soil data, varieties data and crop cultivation and harvest data, which provided data-driven function for computing model components of potential output. The output database was made up of potential coefficient data, total potential data and thematic evaluation data, which provided data-driven function for model verification and potential distribution evaluation model component.

Crop potential output computing component was made up of the standard parameter library of crop species, regional parameter library of crop species, metadata and model file library.

Driven by the input database, system generated a set of crop potential output model views. Driven by model management, models were imported and exported, and model parameters were adjusted according to local conditions. Driven by the suitable potential model, crop potential output coefficients and total potential output were calculated and exported into the output database. Driven by crop potential evaluation components, crop potential output thematic analysis and the credibility of potential models were realized. Fig.1. shows the architecture of crop potential output model system.

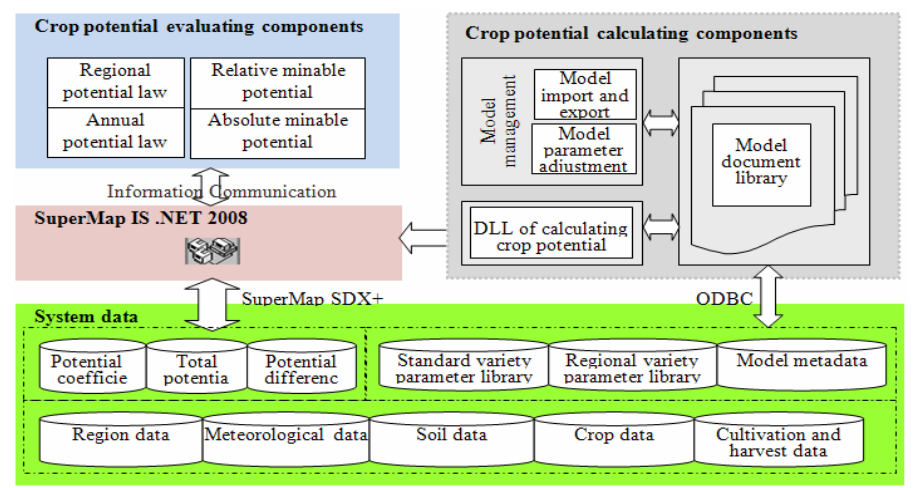

Fig. 1. System architecture

\subsection{Design of Crop Potential Model Component}

Crop has different growth characteristics and crop potential output was affected by multiple factors. So, the paper used computer technology to calculate crop potential and input all relevant data, such as climate conditions, soil conditions, crop species 
and other data and parameters. Based on the analysis to wheat, maize, cotton and other potential output model, the constructed model component should have the characteristics of abstract and polymorphism, which could cover a variety of crop potential output model. The paper built the corresponding model components by using componentware technology to screen the difference in the calculation process. In crop model components, the sub-model should be refined as far as possible to build an autocephalous atom component.

In addition, model components' integration and expansion should be considered with other agricultural production system, so the interface technology was used to highly abstract model components and build a unified data interface. Crop potential output model system was made up of various types of crop model components, such as crop species interface, potential computing interface, and potential evaluation interfaces.

Design of Crop Potential Model Interface. According to the empirical models of crop potential output, system model components consisted of the interface and class of photosynthetic potential, temperature potential, climate potential, soil potential, and socio-economic potential. In view of photosynthesis potential associated with solar radiation, solar radiation object was designed as a attribute in photosynthetic potential class, and solar radiation class was also associated with photosynthetic potential class. Crop potential output interface was provided from crop potential output model system, and was responsible for calculating photosynthesis, temperature, climate, soil and socio-economic potential coefficient and total potential. Photosynthesis, temperature, climate, soil and society sub-interfaces and classes were derived from

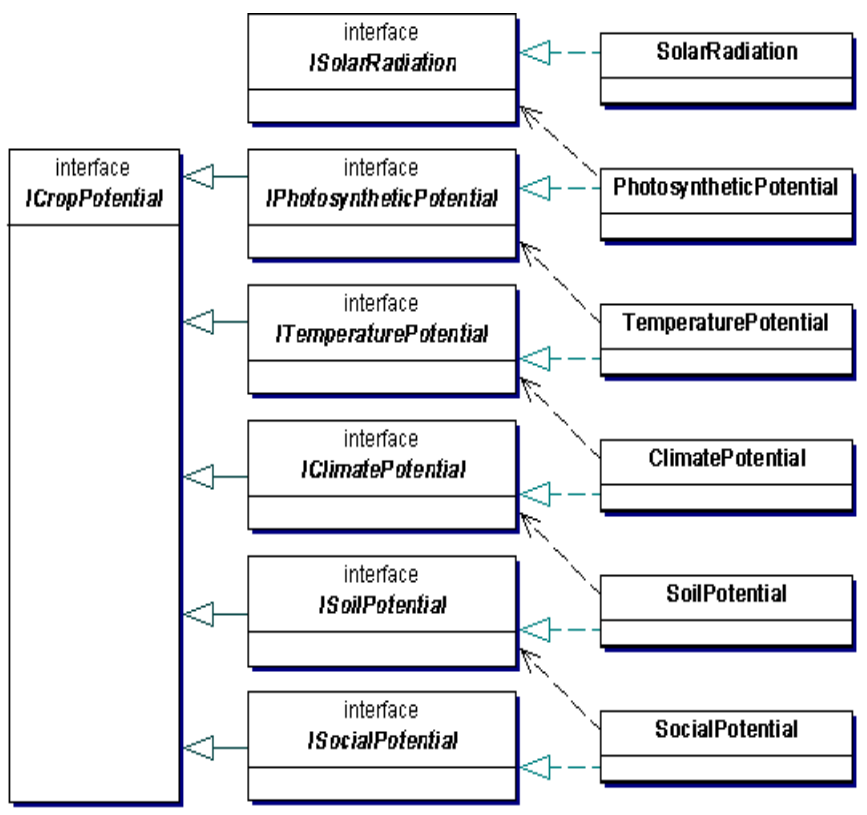

Fig. 2. Model component interface of crop potential output 


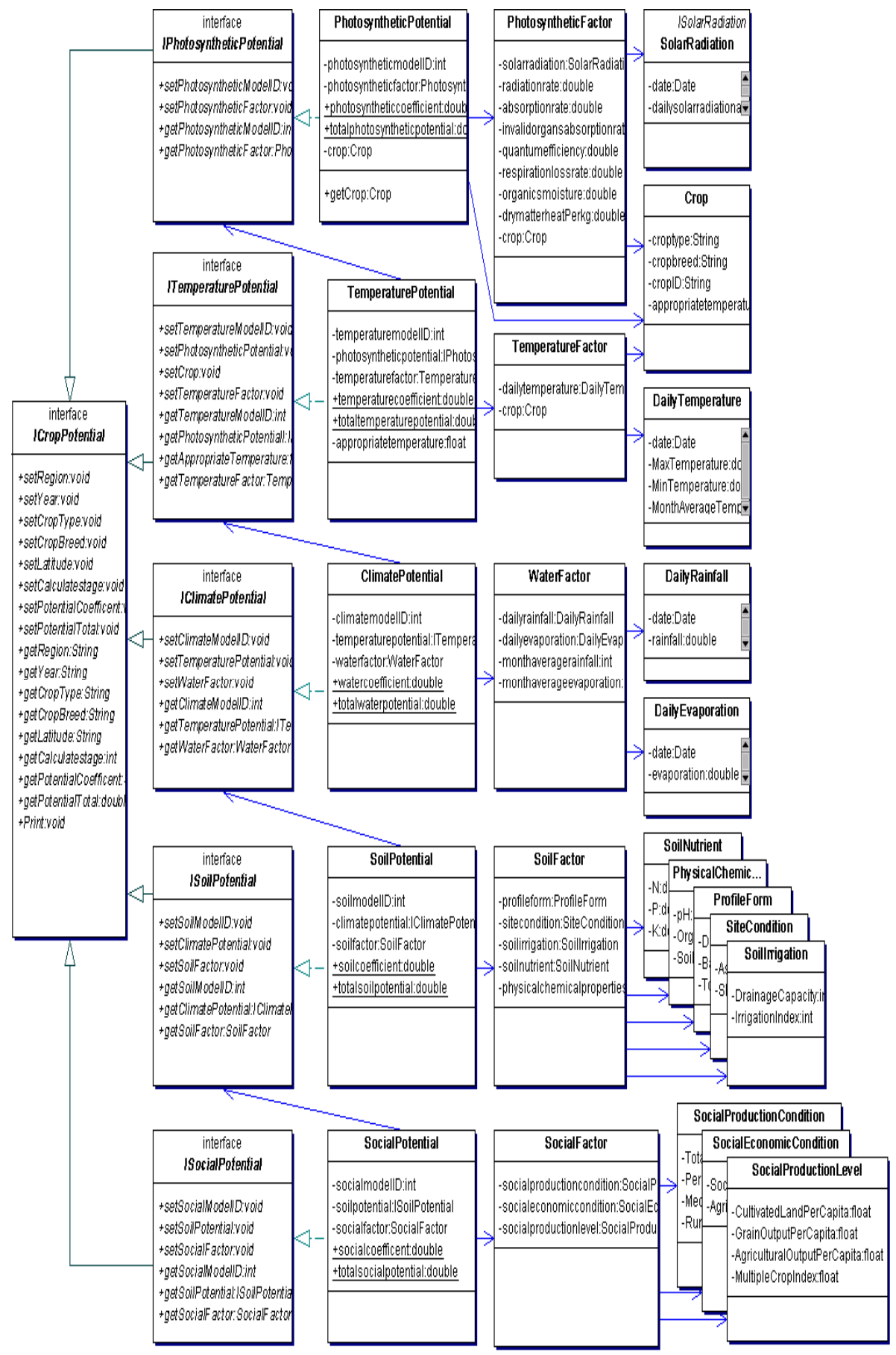

Fig. 3. The integrated potential model components 
crop potential output interface. Five classes at all levels of the potential output, potential coefficient and the total amount were derived from the corresponding subinterface.

Taking into account the relationship among models and the integration of different models at all levels, interface technology was used to design coupled models loosely in crop potential output model system. Fig.2. shows the model component interface of crop potential output. For example, photosynthetic potential output class was associated with solar radiation interface, and solar radiation class was integrated into the photosynthetic potential class to obtain solar radiation. Solar radiation class realized the interface, regardless of which type of solar radiation class for calculating the total solar radiation, as well as temperature potential class, climate potential class, soil potential class and social potential class. Using interface abstraction mechanism could greatly improve the scalability of crop potential output model system.

Design and Integration of Crop Potential Model Subcomponent. Crop potential model subcomponents included photosynthetic potential component, temperature potential component, climate potential component, soil potential and social potential component. Taking into account the characteristics of multi-crop and multi-model, interface technology was used to effectively integrate all levels of models for improving model system's scalability and maintainability. Fig.3. shows the integrated potential model components.

\section{System Implementation and Application}

\subsection{System Implementation}

The container component of crop potential model system provided the potential model interface for different crop production management and dynamic function calls, imported all kinds of crop potential models, and retained the further capacity for expansion interfaces to integrate other agriculture information system.

Taking wheat and maize production in Henan Province as example, the meteorological data, soil data and crop cultivation and harvest data of all cities in Henan Province were inputted into the model system, the potential yield of wheat and maize was quantitatively estimated through the calculating model subsystem, and the potential distribution of wheat and maize at all cities was qualitatively analyzed through the evaluation model subsystem, which provided the decision-making and technical support for building the core area of crop in Henan Province.

\subsection{System Application}

The system has been applied to crop production management in Henan Province. The system is running well, and effectively estimates and predicts crop potential output. Fig.4. shows the interface of crop potential calculation. Fig.5. shows the interface of crop potential analysis. 


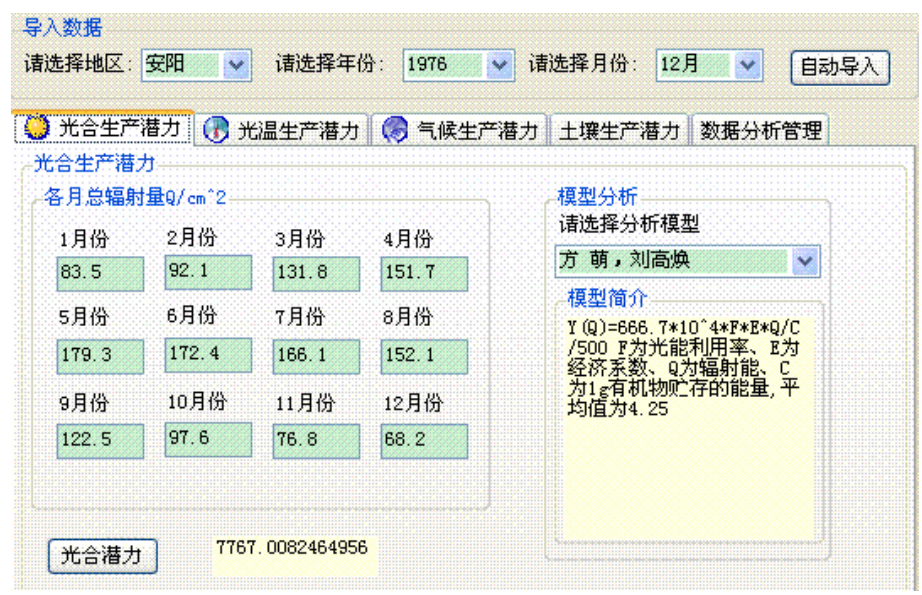

Fig. 4. The interface of crop potential calculation

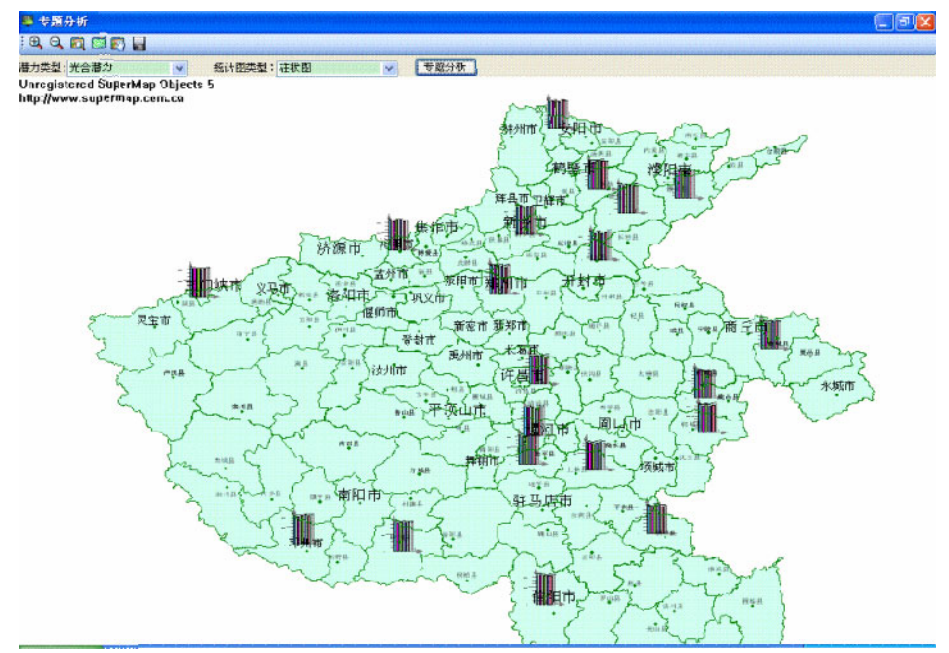

Fig. 5. The interface of crop potential analysis

\section{Conclusion}

Crop potential output model system was built by using componentware technology based on GIS, and crop potential was calculated and quantitatively analyzed. The results show that the system has the characteristics of wide applicability and good independence, which provides ADM and technical support for construction of major grain-producing areas, crop production management and potential mining. The model system could be extended to other crop potential calculation and also combined with other software system, such as soil productivity evaluation system, agriculture fertilization expert system and crop production early warning system. 
It is necessary to strengthen the function of crop potential model management, improve the component management and enrich the model types of crop potential practically to increase productivity in the model system, such as crop growth simulation models, nutrient dynamics and balanced fertilization models and crop output benefit models.

Acknowledgments. This work is supported by "Eleventh Five-Year" national scientific and technological support and major project plan: "High-yield Crop Science and Technology Engineering" (Contract Number: 2006BAD02A07-4), specific industry research sponsored by ministry of agriculture (Contract Number: 20083028) and "863" plan(Contract Number: 2006AA10Z271). Sincerely thanks are also due to the National Engineering Research Center of Wheat for providing the data for the study.

\section{References}

1. Gao, L.Z., Jin, Z.Q., Huang, Y., Chen, H.: The Combination of Crop Simulation and Cultivation Optimization Theory-RCSODS. Crops 3, 4-7 (1994)

2. Zhao, C.J., Wu, H.R., Yang, B.Z., Sun, X., Wang, J.H., Gu, J.Q.: Development Platform for Agricultural Intelligent System Based on Techno-Componentware Model. Trans. CSAE 2, 140-143 (2004)

3. Cao, W.X., Zhu, Y.: Crop Management Knowledge Model. China Agriculture Press, Beijing (2005)

4. Xi, L., Ma, X.M., Li, F.C., Liu, H.B., Ren, Y.N., Li, Y.H.: Design and Implementation of Crop Growth Simulation System Based on Techno-Componentware Model. Jour. Hen. Agri. Uni. 3, 317-320 (2005)

5. Zhang, H., Li, F.C., Ma, X.M., Gao, R., Xia, B., Lu, Z.M.: Design and Realization of GISBased County Testing Soil for Wheat Formulated Fertilization System. Jour. Hen. Agri. Uni. 5, 566-569 (2008)

6. Zhu, Y., Cao, W.X., Wang, S.H., Pan, J.: Application of Soft Component Technology to Design of Intelligent Decision-Making System for Crop Management. Trans. CSAE 1, 132-136 (2003)

7. Wei, K.X.: Henan TuRang Dili. Henan Science and Technology Press, Henan (1995)

8. Zhang, H., Xi, L., Xu, X., Gao, R., Ma, X.M., Yin, J.: Evaluation System of Wheat Natural Potential Productivity at County Scale Based on GIS. Trans. CSAE 12, 198-205 (2009)

9. Zhou, Z.G., Meng, Y.L., Cao, W.X.: Knowledge Model and GIS-based Crop Potential Productivity Evaluation. Scie. Agri. Sini. 6, 1142-1147 (2005)

10. Bai, L.P., Chen, F.: Status and Evaluations on Research of Crop Production Potential in China and Abroad. Crops 1, 7-9 (2002)

11. Xu, C.D., Gao, X.F.: Crop Productivity Potential Model Applied in China. Jour. Arid. Land. Res. Envi. 6, 108-112 (2003)

12. Gu, D.Y., Liu, J.G., Yang, Z.Q., Yin, J.: Reviews on Crop Productivity Potential Researches. Agri. Rese. Arid. Area. 5, 89-94 (2007)

13. Zheng, G.Q.: MAIZESIM-A Model to Simulate Maize Growth and Development. Nanjing Agricultural University, Nanjing (1999)

14. Huang, B.W.: Natural Conditions and Crop Production-Photosynthetic Potential. Science Press, Beijing (1985) 
15. Agricultural Climate Resources in China, http://sky.hzau.edu.cn/BookPath/index.htm

16. Mao, Y.L.: Estimation of Potential Productivities of Main Crops in the Coastal Area of Fujian Province. Jour. Fu. Agri. Fore. Uni.: Natu. Scie. Edit. 2, 255-258 (2002)

17. Shao, X.M., Liu, C.L.: A Study on the Agricultural Potential Land Productivity in the Northwestern Shandong. Chinese Jour. Agro. 4, 5-10 (2004)

18. Wang, H.B., Xu, H., Huo, X.L., Ren, J.Q.: A Study on the Calculating Method of the Soil Effective Coefficient in the Alluvial Plain. Jour. Agri. Uni. Hebei. 4, 53-56 (2002) 